\title{
Propagation of Cosmic Rays and their Secondaries in the Intracluster Medium
}

\section{Saqib Hussain*}

Institute of Astronomy, Geophysics and Atmospheric Sciences (IAG), University of São Paulo (USP), São Paulo, Brazil

E-mail: s.hussain@usp.br

\section{Rafael Alves Batista}

Institute of Astronomy, Geophysics and Atmospheric Sciences (IAG), University of São Paulo (USP), São Paulo, Brazil

E-mail: rafael.abeusp.br

\section{Elisabete Maria de Gouveia Dal Pino}

Institute of Astronomy, Geophysics and Atmospheric Sciences (IAG), University of São Paulo (USP), São Paulo, Brazil

E-mail: dalpinodiag.usp.br

\begin{abstract}
We present results of the propagation of high-energy cosmic rays (CRs) in the intracluster medium (ICM). To this end, we employ three-dimensional cosmological magnetohydrodynamical simulations of the turbulent intergalactic medium to explore the propagation of CRs with energies between $10^{14}$ and $10^{19} \mathrm{eV}$. We study the interaction of test particles with this environment and compute the associated fluxes of CRs and neutrinos using a Monte Carlo simulations.
\end{abstract}

36th International Cosmic Ray Conference -ICRC2019-

July 24th - August 1st, 2019

Madison, WI, U.S.A.

\footnotetext{
*Speaker.
} 


\section{Introduction}

Cosmic rays (CRs) are produced via shocks and turbulent acceleration processes inside galaxies, specially the active ones, and re-accelerated by similar processes in the more diffuse regions of the intracluster medium (ICM), like in shocks induced by cluster mergers, filaments, relics and haloes [1]. CRs with energies $E \gtrsim 8 \times 10^{18} \mathrm{eV}$ likely have an extragalactic origin [2] (for a review see ref. [3]). However, it is not yet clear at which energy there is a transition between galactic and extragalactic CRs. At $E \lesssim 10^{17} \mathrm{eV}$ are believed to have a galactic origin, since their Larmor radii are comparable with the size of the Milky Way (see ref. $[4,5]$ for a review on galactic cosmic rays). These CRs may also be confined within clusters; that is the topic of our investigation.

The energy-loss time of CRs in the ICM makes clusters efficient sites for their storage and production of neutrinos and gamma rays $[6,7,8,9,10]$. The CRs present in the ICM will necessarily produce secondary particles through their interaction with thermal protons and local photon fields. Thus, their properties can be constrained by radio and gamma-ray observations [6, 11, 12].

The $\mathrm{x}$-ray emission from clusters is due to the diffuse intracluster gas, which is at temperatures $T_{g} \approx 10^{6}-10^{8} \mathrm{~K}[1]$. At such temperatures, the primary emission process for a gas composed mainly of hydrogen is thermal Bbemsstrahlung (free-free) emission.

Here we perform detailed simulations of CR propagation in the ICM. This article is structured as follows: In section 2 we present the magnetohydrodynamical (MHD) simulations of structure formation employed. In section 3 we describe the methods used to perform the CR propagation in the ICM. The results are discussed in section 4, followed by the conclusions in section 5 .

\section{MHD simulation}

We are interested in studying the propagation of CRs in the ICM. For that, we use the largescale cosmological MHD simulations performed by Dolag et al. [13, 14], shown in fig. 1. These 3D-MHD simulations were obtained using the SPH (smooth particle hydrodynamics) code GADGET $[15,16]$. They cover a volume of $\sim(140 \mathrm{Mpc})^{3}$. We select two clusters from this large volume. From that, we obtain the magnetic field, gas density, and temperature.

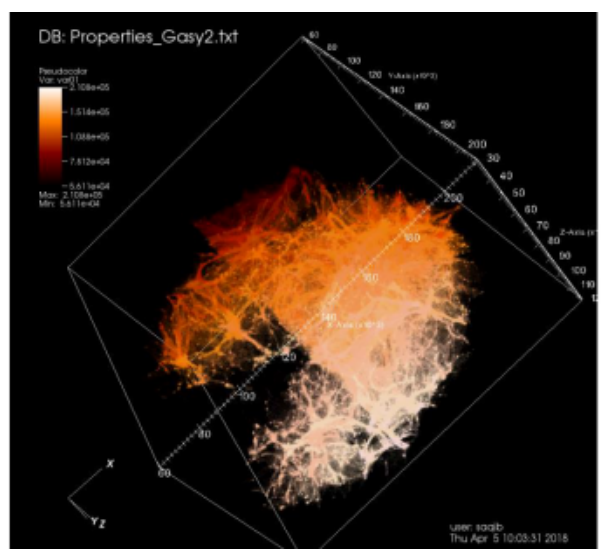

Figure 1: Large-scale distribution of matter, including filaments and clusters, in a domain of 240 $\mathrm{Mpc}^{3}$ [14] 
To perform the CR propagation in the ICM, we also need the distribution of photons within the clusters. We assume that the cluster is filled with a photon field resulting from thermal Bremsstrahlung. The temperatures in the hot ICM range from about $10^{6} \mathrm{~K}$ to $10^{8} \mathrm{~K}$. Thus, these thermal ultraviolet and $\mathrm{x}$-ray photons are the main target fields for CR interactions.

In fig. 2 we show maps for the magnetic field, gas density, temperature, and thermal photon density for one of the clusters.

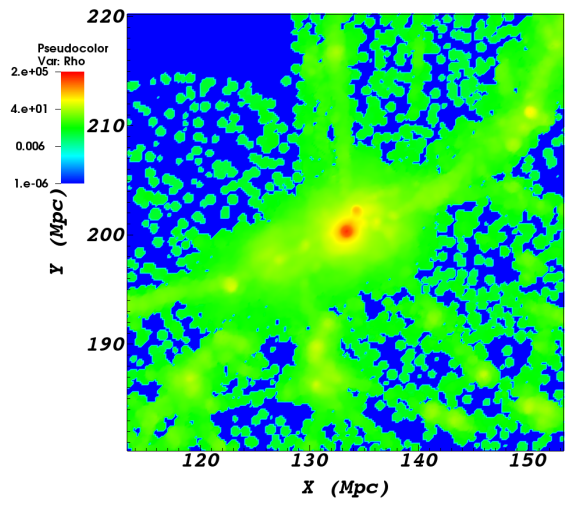

(a) Gas density $\left[\mathrm{g} \mathrm{cm}^{-3}\right]$

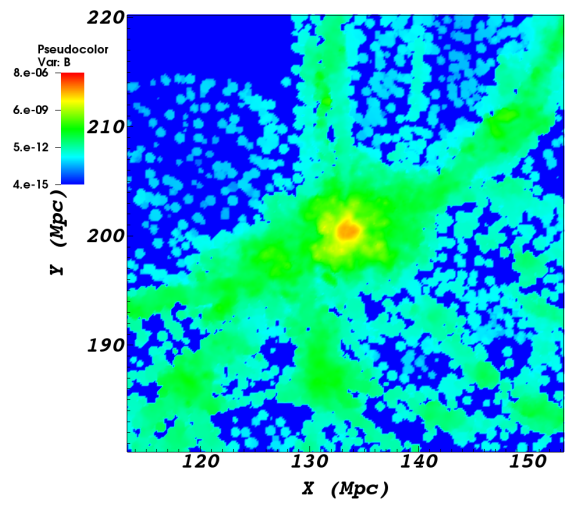

(c) Magnetic field [G]

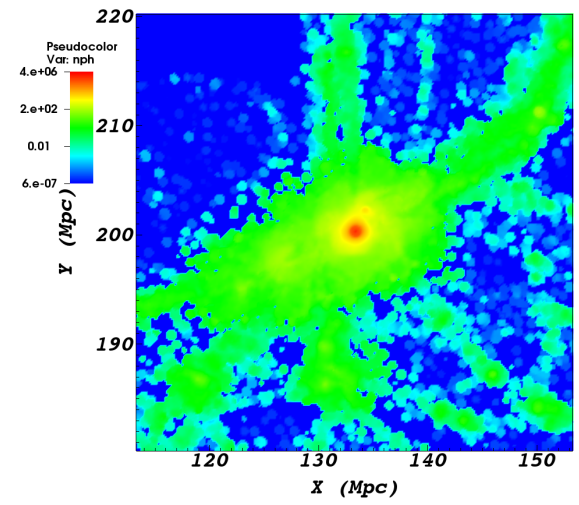

(b) Photon density $\left[\mathrm{cm}^{-3}\right]$

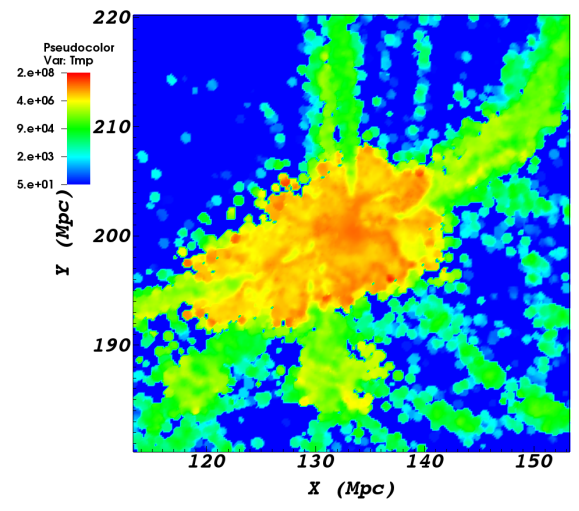

(d) Temperature $[\mathrm{K}]$

Figure 2: This figure shows the maps of gas density (upper left panel), photon density (upper right), magnetic field (lower left), tamperature (lower right) for one of the clusters.

The photon density in different regions of the cluster is not constant. For this reason, we divided the cluster in five regions: $0<r<100 \mathrm{kpc}, 100<r<500 \mathrm{kpc}, 500<r<1000 \mathrm{kpc}$, $1000<r<1500 \mathrm{kpc}, 1500<r<2000 \mathrm{kpc}$. We then estimate the average photon density in each one of them. In fig. 3 we show the average photon field inside the two clusters.

\section{Simulations of CR Propagation}

Cosmic rays are deflected by magnetic fields. Their transport in turbulent media is very complex and requires a detailed modeling to describe the stochastic variations of the pitch angle of the 


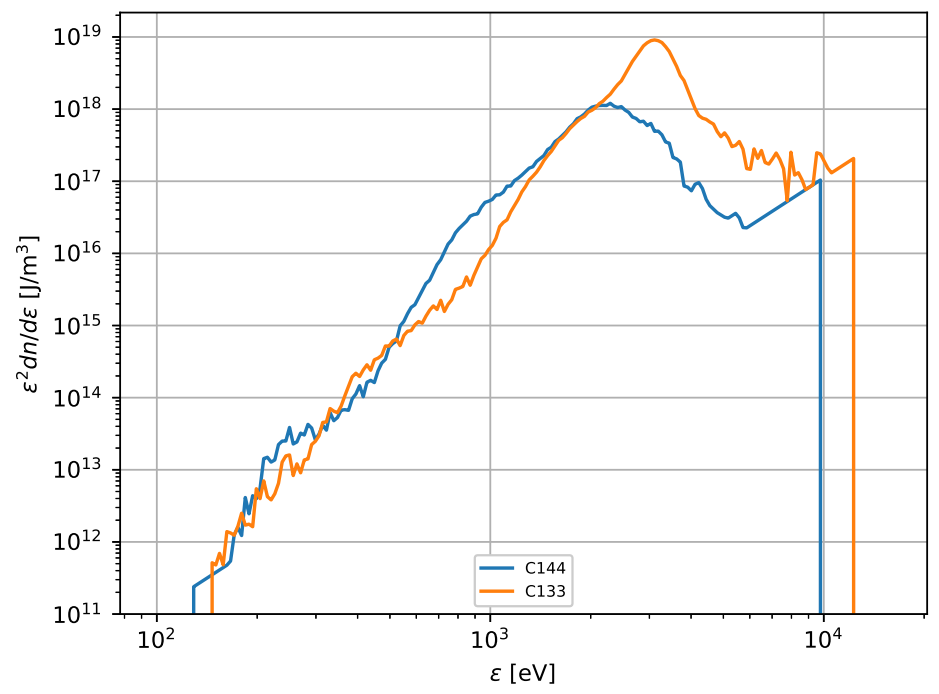

Figure 3: Density of the photon field for the two clusters.

particles in the magnetic fields. For the energies and magnetic field strengths relevant for this study, the propagation of CRs is diffusive, which leads to scatterings that can be interpreted by means of diffusion coefficients.

We model the propagation of CRs in the ICM using the CRPropa 3 code [17]. We also employ the module presented in ref. [18], which solves the transport equation using stochastic diffusion equation.

We assume a flat (in logarithmic scale) spectrum for the CR injection, with energies between $10^{14}$ and $10^{19} \mathrm{eV}$. We take into account all relevant photohadronic and photonuclear processes, namely photopion production, photodisintegration, and Bethe-Heitler pair production. We also consider the adiabatic energy losses due to expansion of the universe.

We consider the injection of protons, nitrogen, and iron nuclei in three different positions in the clusters: the central region, $200 \mathrm{kpc}$ and $1 \mathrm{Mpc}$ away from the centre. These choices correspond to different scenarios of CR acceleration in clusters; they can be accelerated by sources near their central regions, or by shocks in their outskirts. The CR fluxes are computed by an observer of size $1 \mathrm{Mpc}$ sphere centred at the cluster centre for all the three injection points of CRs. Neutrino fluxes are also calculated by the same observer configuration but only for the central region.

\section{Results}

We first study how CR spectrum depends on the position where the CRs are injected in the cluster. This is shown in figure 4 for proton, nitrogen, and iron primaries.

From fig. 4 one can see that the injected spectrum $\left(E^{-1}\right)$ is clearly modified, due to the interactions with thermal photons in the cluster. Higher-energy CRs can escape the cluster, whereas the lower energy ones remain trapped within it, suffering continuous electromagnetic cascading thus explaining the decrease of the flux of CRs at energies $E \lesssim 10^{16} \mathrm{eV}[19]$. 


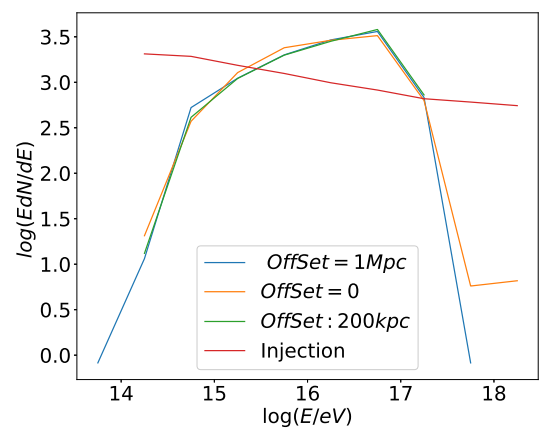

(a) Iron

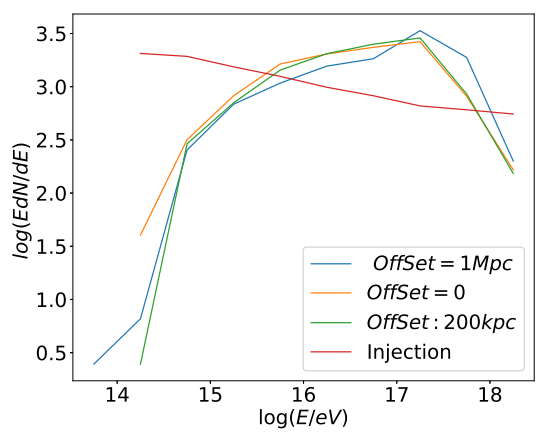

(c) Nitrogen

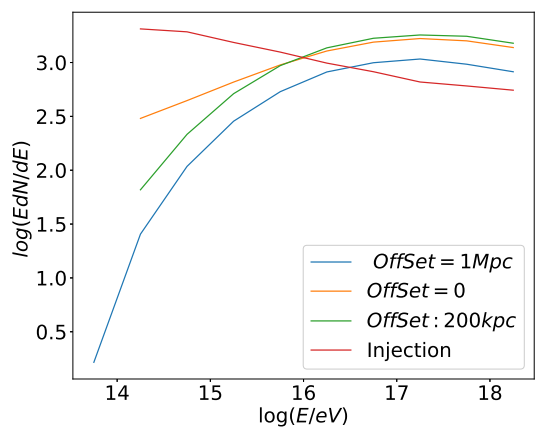

(e) Proton

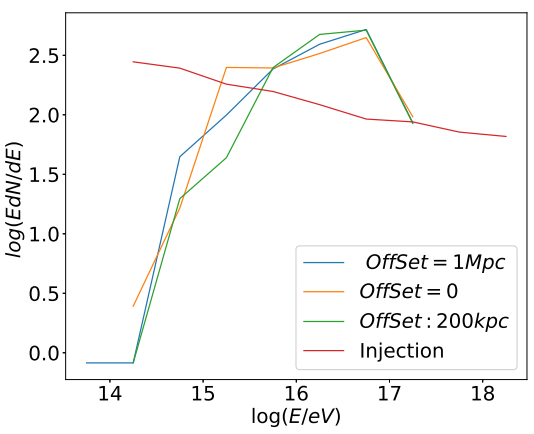

(b) Iron

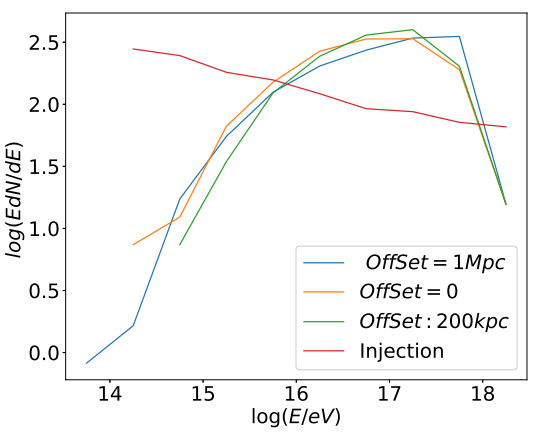

(d) Nitrogen

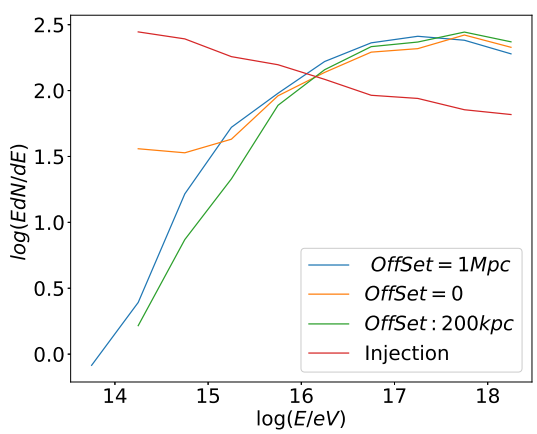

(f) Proton

Figure 4: Spectrum (in arbitrary units) of CRs for cluster 1 (left) and cluster 2 (right column). We assume different compositions at injection: iron (upper panels), nitrogen (middle), and proton (lower). The lines in each plot correspond to: the injected spectrum (red), the flux for a CR source in the centre of the cluster (orange), at $200 \mathrm{kpc}$ (green) and $1 \mathrm{Mpc}$ (blue) away from the centre.

Even very small magnetic field fluctuations in the ICM, can be enough for the confinement of the CRs in the very large volume of galaxy clusters for a time-period comparable to the age of clusters themselves [1]. This magnetic horizon effect effectively limits the energy of CRs that can escape clusters, suppressing the contribution of the lower-energy ones.

We also compute the neutrinos stemming from CR interactions in the ICM. These are shown 
in figure 5. It is clear from fig. 5 that most of the neutrinos produced at $E \sim 1 \mathrm{PeV}$ originate from

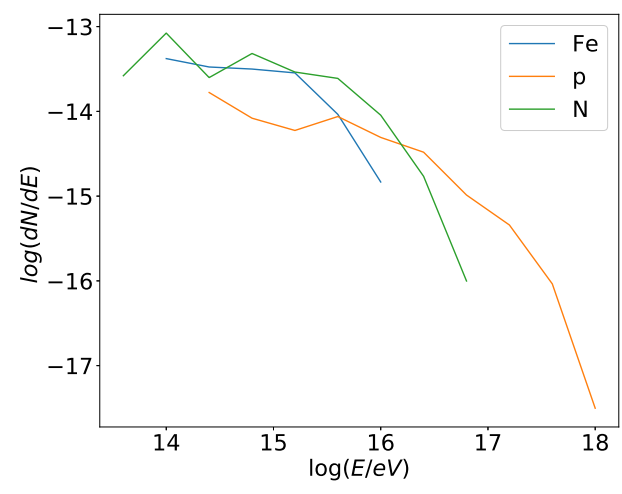

(a) Cluster 1

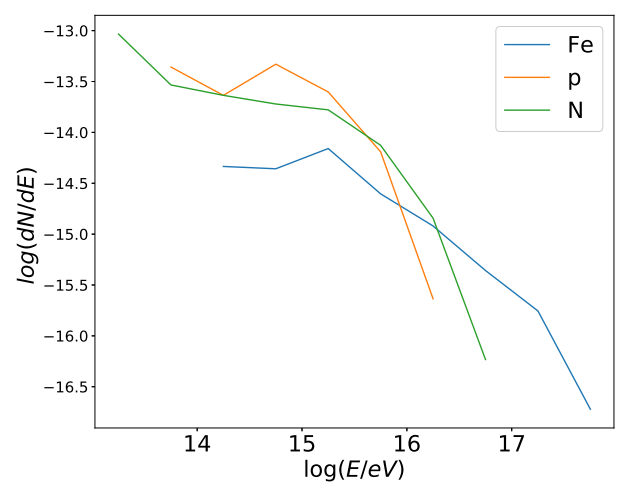

(b) Cluster 2

Figure 5: Spectrum of neutrinos (in arbitrary units) for cluster 1 (left) and cluster 2 (right panel), for different assumptions on the composition of cosmic-ray primaries.

beta decays of nuclei produced in the photodisintegration chain.

\section{Discussion and Conclusions}

We performed a detailed analysis of CR propagation in clusters of galaxies using 3D-MHD simulations. By combining high-resolution models of clusters, obtained from cosmological simulations, with a Monte Carlo method for radiative transfer, we intended to provide a complete description for high-energy emission by galaxy clusters.

We find that the CRs with energy $E \lesssim 10^{16} \mathrm{eV}$ cannot escape the innermost regions of clusters. We note that this upper limit is more constrained than the one we obtained previously when neglecting the CR interactions with the background thermal photon field $\left(E \lesssim 10^{17} \mathrm{eV}\right)$ [19]. In this case, CRs are confined within the cluster for a time comparable to the age of the universe. These results are expected as the the Larmor radius of a $10^{16} \mathrm{eV} \mathrm{CR}$ in a magnetic field $B=\mu \mathrm{G}$ is $R_{L} \sim 0.01 \mathrm{kpc}$, which is much smaller than the size of the cluster.

In this work, we have not considered the interaction between CRs and the gas in the cluster. This is, however, a well-known channel for neutrino and gamma-ray production (see e.g. [8, 9]).

During their confinement in the ICM, CRs produce secondary particles like pions, whose decays $\left(\pi^{0} \rightarrow 2 \gamma, \pi^{+} \rightarrow \mu^{+}+v_{\mu}, \pi^{-} \rightarrow \mu^{-}+\bar{v}_{\mu}\right)$ naturally result in photons and electrons. Hence, the confinement of CRs in the ICM can produce a significant amount of gamma rays. We have not yet studied the production of gamma rays, nor the synchrotron emission in the ICM. We plan on extending our work to obtain a detailed gamma-ray emission model. This may be probed with imaging air Cherenkov telescopes such as the upcoming Cherenkov Telescope Array (CTA), which will observe clusters with unprecedented precision [20].

Clusters are well-known reservoirs of CRs, and efficient sites for high-energy neutrino production. Presently, only one source of high-energy neutrino has been observed [21], but we expect more to be found with experimental efforts such as the IceCube Neutrino Observatory [22], and the 
planned Giant Radio Array for Neutrino Detection (GRAND) [23]. In particular, if the diffuse flux of cosmogenic neutrinos that might contaminate the signal is low, as suggested in [24], neutrinos from clusters are even more likely to be detected.

In summary, galaxy clusters are unique environments for high-energy particle production, including neutrinos and gamma rays, due to the photohadronic, photonuclear, and hadronuclear processes taking place in its interior. Because of its magnetic field, the confinement of CRs for long periods of time enhances the interaction rates, increasing the production of secondaries. The integrated contribution of all clusters in the universe imply that they may respond for a sizeable fraction of the diffuse gamma-ray and neutrino backgrounds.

\section{Acknowledgements}

This work is supported by the São Paulo Research Foundation (FAPESP) grants \#2017/128284 and \#2013/10559-5, and by CNPq grant (\#306598/2009-4).

\section{References}

[1] G. Brunetti \& T.W. Jones, Int. J. Mod. Phys. D 23 (2014) 1430007.

[2] Pierre Auger Collaboration 2017, Science 357 (2017) 1266.

[3] R. Alves Batista et al., Front. Astron. Space Sci. 6 (2019) 23.

[4] P. Blasi, Astron. Astrophys. Rev. 21 (2013) 70.

[5] E. Amato \& P. Blasi., Adv. Space Res. 62 (2018) 2731.

[6] V. S. Berezinsky, P. Blasi, V. S. Ptuskin, Astrophys. J. 487 (1997) 529.

[7] C. Rordorf, D. Grasso, K. Dolag, Astropart. Phys. 22 (2004) 167.

[8] K. Kotera et al., Astrophys. J. 707 (2009) 370.

[9] K. Murase \& J. Beacom. J. Cosmol. Aastropart. Phys. 02 (2013) 028.

[10] K. Fang \& A. V. Olinto, Astrophys. J. 828 (2016) 37.

[11] B. Wolfe et al., Astrophys. J. 675 (2008) 156.

[12] T. M. Yoast-Hull et al., Astrophys. J. 768 (2013) 53.

[13] K. Dolag et al., JETP Lett. 79 (2004) 583.

[14] K. Dolag et al., J. Cosmol. Astropart. Phys. 01 (2005) 009.

[15] V. Springel, N. Yoshida, S. D. M. White, New Astron. 6 (2001) 51.

[16] V. Springel V., Mon. Not. R. Astron. Soc. 364 (2005) 1105.

[17] R. Alves Batista et al., J. Cosmol. Astropart. Phys. 05 (2016) 038.

[18] L. Merten et al., J. Cosmol. Astropart. Phys. 06 (2017) 046.

[19] R. Alves Batista, E. M. de Gouveia Dal Pino, K. Dolag, S. Hussain, Proceedings IAU Focus Meeting 8: New Insights in Extragalactic Magnetic Fields (2019), in press arXiv:1811.03062

[20] CTA Consortium. "Science with the Cherenkov Telescope Array", World Scientific (2019). 
[21] IceCube Collaboration et al., 361 (2018) eaat1378.

[22] IceCube Collaboration, Phys. Rev. Lett. 111 (2013) 021103.

[23] GRAND Collaboration, Science China: Phys., Mech. \& Astron. 63 (2020) 219501.

[24] R. Alves Batista et al., J. Cosmol. Astropart. Phys. 01 (2019) 002. 